Original paper

\title{
The influence of respiratory motion on dose distribution in accelerated partial breast irradiation using volumetric modulated arc therapy
}

\author{
Ryohei Yamauchi *, Norifumi Mizuno, Tomoko Itazawa, Jiro Kawamori \\ Department of Radiation Oncology, St. Luke's International Hospital, Tokyo, Japan
}

\section{A R T I C L E I N F O}

\section{Keywords:}

Breast cancer

Accelerated partial breast irradiation

Intensity modulated radiation therapy

Respiratory motion

\begin{abstract}
A B S T R A C T
Purpose: Accelerated partial breast irradiation (APBI) is alternative treatment option for patients with early stage breast cancer. The interplay effect on volumetric modulated arc therapy APBI (VMAT-APBI) has not been clarified. This study aimed to evaluate the feasibility of VMAT-APBI for patients with small breasts and investigate the amplitude of respiratory motion during VMAT-APBI delivery that significantly affects dose distribution.

Methods: The VMAT-APBI plans were generated with $28.5 \mathrm{~Gy}$ in five fractions. We performed patient-specific quality assurance using Delta ${ }^{4}$ phantom under static conditions. We also measured point dose and dose distribution using the ionization chamber and radiochromic film under static and moving conditions of 2, 3 and $5 \mathrm{~mm}$. We compared the measured and calculated point doses and dose distributions by dose difference and gamma passing rates.

Results: A total of 20 plans were generated; the dose distributions were consistent with those of previous reports. For all measurements under static conditions, the measured and calculated point doses and dose distributions showed good agreement. The dose differences for chamber measurement were within $3 \%$, regardless of moving conditions. The mean gamma passing rates with $3 \% / 2 \mathrm{~mm}$ criteria in the film measurement under static conditions and with $2 \mathrm{~mm}, 3 \mathrm{~mm}$, and $5 \mathrm{~mm}$ of amplitude were $95.0 \pm 2.0 \%, 93.3 \pm 3.3 \%, 92.1 \pm 6.2 \%$ and $84.8 \pm$ $7.8 \%$, respectively. The difference between $5 \mathrm{~mm}$ amplitude and other conditions was statistically significant. Conclusions: Respiratory management should be considered for the risk of unintended dose distribution if the respiratory amplitude is $>5 \mathrm{~mm}$.
\end{abstract}

\section{Introduction}

Breast cancer is the most common cancer in women, with an estimated worldwide incidence of 2.1 million cases in 2018 [1]. Breastconserving surgery followed by whole breast radiotherapy is the current standard of care for patients with early stage breast cancer. Adjuvant radiation therapy is typically employed for breast cancer to reduce the risk of local recurrence and increase overall survival [2,3]. Accelerated partial breast irradiation (APBI) is an alternative treatment option for patients with early stage breast cancer. Several trials, including RAPID [4,5], the National Surgical Adjuvant Breast and Bowel Project, the B-39/Radiation Therapy Oncology Group (RTOG) 0413 [6,7], the Florence trial [8] and the ACCEL trial [9], are currently investigating the potential of external beam APBI to reduce the dose to normal tissues and improve cosmesis thanks to the decreased volume of breast tissue treated. Previous studies have reported that APBI with intensity modulated radiation therapy (IMRT) or volumetric modulated arc therapy (VMAT) increases dose homogeneity and conformity in the target area compared with conventional radiotherapy $[10,11]$ and, as a result, further improves cosmesis $[8,9]$. Kosaka et al. reported that the conformity of the dose distribution for patients with small breasts is less favorable than for those with large breasts. [12] Studies have reported that the radiation dose to the normal mammary gland might be excessive when the surgical cavity is relatively large compared with the breast size $[12,13]$. The breast size of Asian women is generally smaller than that of Europeans and Americans, and, therefore, the dosimetric evaluation of APBI with VMAT (VMAT-APBI) and the feasibility of VMATAPBI for patients with small breasts need to be clarified.

Respiratory motion during breast IMRT has also been investigated. The dosimetric advantage of IMRT is degraded by the interplay between multileaf collimator movement and intrafraction organ motion $[14,15]$. Studies have reported that the interplay effect can be reduced through

\footnotetext{
* Corresponding author at: Department of Radiation Oncology, St. Luke's International Hospital, 9-1 Akashi-cho, Chuo-ku, Tokyo 104-8560, Japan.

E-mail address: yamauchi.ryouhei@gmail.com (R. Yamauchi).
} 
the use of multiple fields and fractionated treatment $[14,16]$. The blurring of dose distributions is also caused by interfractional organ motion and setup error. Task Group 76 of the American Association of Physicists in Medicine (AAPM) recommended considering respiratory motion management (breath holding, respiratory gating, and dynamic tumor tracking techniques) if a $>5 \mathrm{~mm}$ range of motion was observed in any direction [17]. Although numerous studies have reported respiratory management for lung and abdominal cancer during radiation therapy, there have been few reports on respiratory motion analysis for patients with breast cancer $[18,19]$, who might have different respiratory characteristics given their potentially better lung function, their relatively younger age, and the fact that women breathe more with their thorax [20]. We analyzed the respiratory motion in a large population of patients with breast cancer to determine the typical respiratory pattern (e.g., amplitude and cycle) during radiation therapy. Based on this respiratory pattern, we investigated the relationship between respiratory motion and the interplay effect in VMAT-APBI.

The aim of this study was to evaluate the feasibility of VMAT-APBI for Asian patients and to investigate the amplitude of respiratory motion during VMAT-APBI delivery that significantly affects dose distribution.

\section{Methods and materials}

\subsection{Planning study for VMAT-APBI}

\subsubsection{Study population}

We pooled 10 patients with early stage breast cancer ( 5 with right breast cancer and 5 with left breast cancer, with a maximum tumor diameter of $3.0 \mathrm{~cm}$ ) treated at our institution from May 2018 to December 2018. The study protocol was approved by our Institutional Review Board (18-R162). The analysis performed for this study consisted of purely dosimetric modeling and was fully independent of the care delivered to each patient.

\subsubsection{Delineation for target and organs at risk}

Computed tomography (CT) simulation was performed with the patient in the supine position with both arms raised above the head via wing-support immobilization (Engineering System Co., Ltd., Nagano, Japan). CT images ( $2.5 \mathrm{~mm}$ thick) were obtained using the GE LightSpeed RT16 CT scanner (GE Healthcare, Waukesha, WI, USA).

Table 1 shows the study patient characteristics and the mean values for each parameter. A total of six 5.6-mm long surgical titanium clips (Peters Surgical, Bobigny, France) were delineated, and the clinical target volume (CTV) was expanded by adding $1.5 \mathrm{~cm}$ margins. The planning target volume (PTV) was defined by adding $0.5 \mathrm{~cm}$ margins to the CTV and cropping the heart and lungs. To evaluate dose coverage at PTV, we created a dose evaluation volume (DEV_PTV) that excluded 3 $\mathrm{mm}$ cropped from the skin surface. The organs at risk (OARs), including the heart, bilateral breasts, bilateral lungs, and skin, were delineated by the radiation oncologist or medical physicist. The uninvolved breast was defined by subtracting DEV_PTV from the ipsilateral breast. These structure definitions agree with the RTOG Breast cancer atlas [21].

\subsubsection{VMAT-APBI planning}

Two medical physicists generated each 10 VMAT-APBI plans (a total of 20 plans with 10 patients) by using a radiation treatment planning system (RayStation, version 6.2; RaySearch Laboratories, Stockholm, Sweden) employing the same dataset. There were no restrictions on planning parameters, such as optimize settings and monitor units. All plans were generated using VMAT with two partial arcs. For the leftsided plans, we set the starting and ending gantry angles between $165^{\circ}$ and $290^{\circ}$ and the arc length within $215^{\circ}$. For the right-sided plans, we set the angles between $35^{\circ}$ and $190^{\circ}$ and the arc length within $215^{\circ}$. Dose calculation was performed by using a collapsed-cone convolution algorithm with heterogeneity correction and a constant $2 \mathrm{~mm}$ calculation grid size. The prescribed dose was $28.5 \mathrm{~Gy}$ in five fractions. The dose received by at least $95 \%$ of the DEV_PTV volume ( $\mathrm{D}_{95 \%}$ ) was set to 28.5 Gy. We employed a 6 MV X-ray linear accelerator (Clinac-iX; Varian Medical Systems) for all plans, with the couch angle held static at $0^{\circ}$ and the control point spacing set at $2^{\circ}$. Because we could not apply all plans, we did not use a pseudo-skin flash strategy using a virtual bolus during the VMAT planning in the present study.

Previous trials have targeted Europeans and Americans women with relatively large physiques and breasts; however, this study investigated the feasibility of the VMAT-APBI treatment plan in Asian women with small physiques. For that reason, we performed the plan optimization using target and OAR dose constraints referred from studies including Europeans and Americans women (Table 2) [6-8,22,23].

\subsubsection{Dosimetric parameters}

The dosimetric parameters for the target and OARs were calculated by recording the percentage volume of the target or organs receiving a dose greater than X Gy $\left(V_{X}\right.$ Gy $)$, the volume receiving a dose greater than $\mathrm{X} \%$ of the prescribed dose $\left(\mathrm{V}_{\mathrm{X} \%}\right)$, and the dose to the percentage and absolute volumes ( $\mathrm{D}_{\mathrm{X} \text { cc }}$ or $\mathrm{D}_{\mathrm{X} \%}$ ). We calculated the homogeneity index (HI) of DEV_PTV using the following equation from the International Commission on Radiation Units and Measurements report 83 recommendation [24]:

$\mathrm{HI}=\frac{D_{2 \%}-D_{98 \%}}{D_{50 \%}}$.

The dose conformity index (CI) for each plan was calculated based on the RTOG [25] definition:

$\mathrm{CI}=\frac{\mathrm{V}_{\mathrm{RI}}}{\mathrm{TV}}$

Table 1

Patient characteristics for the planning study.

\begin{tabular}{|c|c|c|c|c|c|c|c|}
\hline \multirow[t]{2}{*}{ No. } & \multirow[t]{2}{*}{ Quadrant of the breast } & \multirow[t]{2}{*}{ BMI, $\mathrm{kg} / \mathrm{m}^{2}$} & \multicolumn{4}{|l|}{ Volume, $\mathrm{cm}^{3}$} & \multirow[t]{2}{*}{ Ratio of DEV_PTV to ipsilateral breast, $\%$} \\
\hline & & & Ipsilateral breast & Contralateral breast & CTV & DEV_PTV & \\
\hline Lt-1 & $\mathrm{BD}$ & 18.8 & 389.8 & 373.5 & 23.5 & 49.9 & 12.8 \\
\hline Lt-2 & $\mathrm{E}$ & 35.2 & 1734.3 & 1576.8 & 57.1 & 104.1 & 6.0 \\
\hline Lt-3 & AC & 24.0 & 802.9 & 873.6 & 41.9 & 82.1 & 10.2 \\
\hline Lt-4 & $\mathrm{C}$ & 20.6 & 588.4 & 675.3 & 39.5 & 76.0 & 12.9 \\
\hline Lt-5 & $\mathrm{C}$ & 28.0 & 1540.9 & 1489.0 & 47.3 & 88.3 & 5.7 \\
\hline Rt-1 & $\mathrm{D}$ & 23.4 & 1016.8 & 1005.5 & 70.5 & 123.9 & 12.2 \\
\hline Rt-2 & $\mathrm{E}$ & 20.3 & 631.3 & 607.9 & 46.0 & 96.8 & 15.3 \\
\hline Rt-3 & $\mathrm{C}$ & 26.3 & 1270.3 & 1312.0 & 56.8 & 105.2 & 8.3 \\
\hline Rt-4 & $\mathrm{C}$ & 20.6 & 675.3 & 588.4 & 45.0 & 86.8 & 12.9 \\
\hline Rt-5 & $\mathrm{C}$ & 25.8 & 804.5 & 844.1 & 65.6 & 117.1 & 14.6 \\
\hline Mean & & 24.3 & 945.4 & 934.7 & 49.3 & 93.0 & 11.1 \\
\hline
\end{tabular}

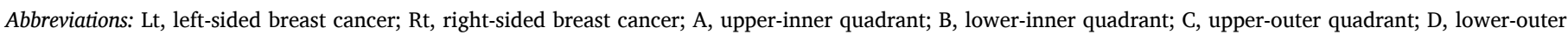
quadrant; E, center; BMI, body mass index. 
Table 2

Dose constraints for the target and normal tissue.

\begin{tabular}{|c|c|c|}
\hline Variables & & Constraint \\
\hline DEV_PTV & $\begin{array}{l}\mathrm{V}_{28.5 \mathrm{~Gy}} \\
\mathrm{~V}_{27 \mathrm{~Gy}} \\
\mathrm{D}_{2 \%} \\
\mathrm{D}_{98 \%}\end{array}$ & $\begin{array}{l}=95.0 \% \\
\geqq 99.0 \% \\
<30.5 \mathrm{~Gy} \\
\geqq 26.0 \mathrm{~Gy}\end{array}$ \\
\hline Uninvolved breast & $\begin{array}{l}V_{50 \%} \\
V_{100 \%}\end{array}$ & $\begin{array}{l}\leqq 40.0 \%(50.0 \%) \\
\leqq 10.0 \%\end{array}$ \\
\hline Contralateral breast & $\mathrm{D}_{2 \mathrm{cc}}$ & $\leqq 1.5 \mathrm{~Gy}(2.0 \mathrm{~Gy})$ \\
\hline Contralateral lung & $\begin{array}{l}V_{20 \text { Gy }} \\
V_{10 \text { Gy }} \\
V_{5 \text { Gy }} \\
V_{1.5 \text { Gy }}\end{array}$ & $\begin{array}{l}\leqq 3.0 \% \\
\leqq 10.0 \% \\
\leqq 20.0 \% \\
\leqq 15.0 \%\end{array}$ \\
\hline Lungs & $\begin{array}{l}V_{20 ~ G y} \\
V_{10 ~ G y} \\
V_{5 \text { Gy }}\end{array}$ & $\begin{array}{l}\leqq 1.0 \%(2.0 \%) \\
\leqq 4.0 \%(5.0 \%) \\
\leqq 8.0 \%(10.0 \%)\end{array}$ \\
\hline Heart & $\begin{array}{l}\mathrm{V}_{1.5 \text { Gy }} \text { (right) } \\
\mathrm{V}_{20 \text { Gy }} \text { (left) } \\
\mathrm{V}_{5 \text { Gy }} \text { (left) } \\
\mathrm{V}_{3 \text { Gy }} \text { (left) } \\
\mathrm{V}_{1.5 \text { Gy }} \text { (left) }\end{array}$ & $\begin{array}{l}\leqq 10.0 \%(15.0 \%) \\
\leqq 1.0 \% \\
\leqq 7.0 \% \\
\leqq 10.0 \% \\
\leqq 40.0 \%\end{array}$ \\
\hline
\end{tabular}

Abbreviations: DEV, dose evaluation volume; PTV, planning target volume.

where $\mathrm{V}_{\mathrm{RI}}$ is the volume receiving $100 \%$ of the prescribed dose and TV is the target volume. The CI defined by RTOG evaluates the dose conformity between the target volume and dose volume; however, this equation is not accounted for the overlap of the volume covered by the prescribed dose and the target volume, and it is uncertain whether the two volumes coincide even if the CI is equal to 1 [26]. Paddick proposed an alternative conformity index [27] building on the criticism of the RTOG conformity index as follows,

Paddick CI $=\frac{\mathrm{TV}_{\mathrm{RI}}}{\mathrm{TV} \times \mathrm{V}_{\mathrm{RI}}}$

where, $\mathrm{TV}_{\mathrm{RI}}$ is the target volume covered by prescribed dose. It is between 0 and 1 , with highest score representing the most conformal plan.

The gradient index (GI) [27] is defined as follows:

$\mathrm{GI}=\frac{\mathrm{V}_{501 \%}}{\mathrm{~V}_{100 \%}}$

where $\mathrm{V}_{\mathrm{X}}$ \% represent the volume receiving a dose greater than $\mathrm{X} \%$ of the prescribed dose. The lower the GI value, the dose fall-off outside the target and better sparing of healthy tissue.

The monitor units and estimated beam-on times were retrieved directly from the beam properties in the radiation treatment planning system.

\subsection{Influence of respiratory motion on dose distribution}

\subsubsection{Analysis of breast respiratory motion}

To evaluate breast respiratory motion, we recorded approximately 1 min of motion tracking during free breathing using the Varian real-time position management (RPM) system (Varian Medical Systems, Palo Alto, CA, USA) for 50 newly selected patients with breast cancer. The RPM infrared box marker was placed on the center of the patient's breast (near the nipple), and the respiratory motion statistics were analyzed by RPM 3-axis tracking data.

\subsubsection{Patient-specific quality assurance under static conditions}

The patient-specific dosimetric quality assurance (QA) of the 20 VMAT-APBI plans was performed using a 3D dose verification system (Delta ${ }^{4}$, ScandiDos, Uppsala, Sweden) under static conditions. The Delta ${ }^{4}$ phantom has silicon diodes that are placed at $5 \mathrm{~mm}$ or $10 \mathrm{~mm}$ intervals on two orthogonal boards. The gamma analysis was performed using Delta ${ }^{4}$ software to compare the measured and calculated dose distributions. We performed the global gamma analysis using the dose difference/distance-to-agreement criteria of $3 \% / 2 \mathrm{~mm}$ for the area receiving $>20 \%$ isodose to assess patient-specific QA. Criteria of 3\%/3 $\mathrm{mm}$ and $2 \% / 2 \mathrm{~mm}$ were also used to assess the gamma passing rates.

\subsubsection{Patient-specific $Q A$ with respiratory motion}

Fig. 1 shows the measurement setup for the breast phantom and the four-axis moving platform. We developed an original breast phantom (Taisei Medical, Osaka, Japan) modeling the average Asian woman's body shape for our study to measure point doses and dose distributions. The breast phantom consists of phantoms equivalent to water, lungs, and bones. This phantom was designed in a way that it could be divided into two parts at the center of its long axis (superior-inferior direction), and a film could be inserted. Furthermore, it provided holes for ionization chamber insertion, and the geometric center of the ionization chamber was designed to match the measuring plane of the film. In this study, the point dose was measured using a mini-type ionization chamber (TM31010, PTW, Freiburg, Germany), and the dose distribution of transversal plane was measured using radiochromic film (Gafchromic EBT3 dosimetry film, Ashland, NJ, USA).

The phantom was mounted on a 4-axis moving platform (4D-KAMUI, Anzai Medical, Tokyo, Japan), which consisted of three orthogonal linear stages for the target (phantom) and a fourth stage for surrogate motion with $20 \mathrm{~ms}$ control intervals [28]. The platform was powered by three computer-controlled independent linear stages $(75 \mathrm{~mm}$ motion range in the anterior-posterior, superior-inferior and lateral directions; $45 \mathrm{~mm} / \mathrm{s}$ maximum velocity; $1 \mathrm{~mm}$ maximum positional accuracy). The platform allows for the simulation of sinusoidal and arbitrary waveforms.

The breast phantom was scanned in the absence of motion using a CT scanner. The images were then imported into the treatment planning system. Ten plans of different 10 patients out of the total 20 plans (see section "VMAT-APBI planning") were selected and were re-calculated on the breast phantom. Because there was no large difference in the plan quality (such as dosimetric parameters, monitor units, gantry angle and beam-on times) due to the difference in the planners, 5 plans of each were randomly selected. We delivered the VMAT-APBI plans to the breast phantom, using patterns for one static condition and three respiratory motion conditions (in this case, 40 datasets $=4$ conditions $\times 10$ plans), and then measured the point doses and dose distributions using the ionization chamber and radiochromic film, respectively. On measuring using the ionization chamber and radiochromic film during respiratory motion, we used the three respiratory waveforms to move the three axes. There were no criteria in terms of beam-on start with respect to motion curve, and irradiation took place in a random manner.

We compared the measured point dose of the isocenter with the calculated dose and determined the dose difference. To eliminate the bias introduced by the difference between the treatment planning system, the results with respect to static condition measurements were also calculated as follows,

Relative dose difference $(\%)=\frac{D_{\text {Resp }}-D_{\text {Static }}}{D_{\text {Static }}} \times 100$

where $D_{\text {Resp }}$ is dose measured under each respiratory condition and $D_{\text {Static }}$ is an average dose measured under static conditions. We scanned the measured films in transmission mode in the same orientation using a resolution of $96 \mathrm{dpi}$ in the 48-bit red-channel color scale. We performed translational and rotational corrections, analyzing all films with OmniPro I'mRT software version 1.7 (IBA dosimetry, Germany). The dose distribution was normalized by the dose near the isocenter after densityto-dose conversion and compared the corresponding measured and calculated dose distributions for the area receiving $>35 \%$ isodose. Subsequently, we performed a global gamma analysis using the criteria of 3\%/2 mm to assess patient-specific film QA. Criteria of 3\%/3 mm and 


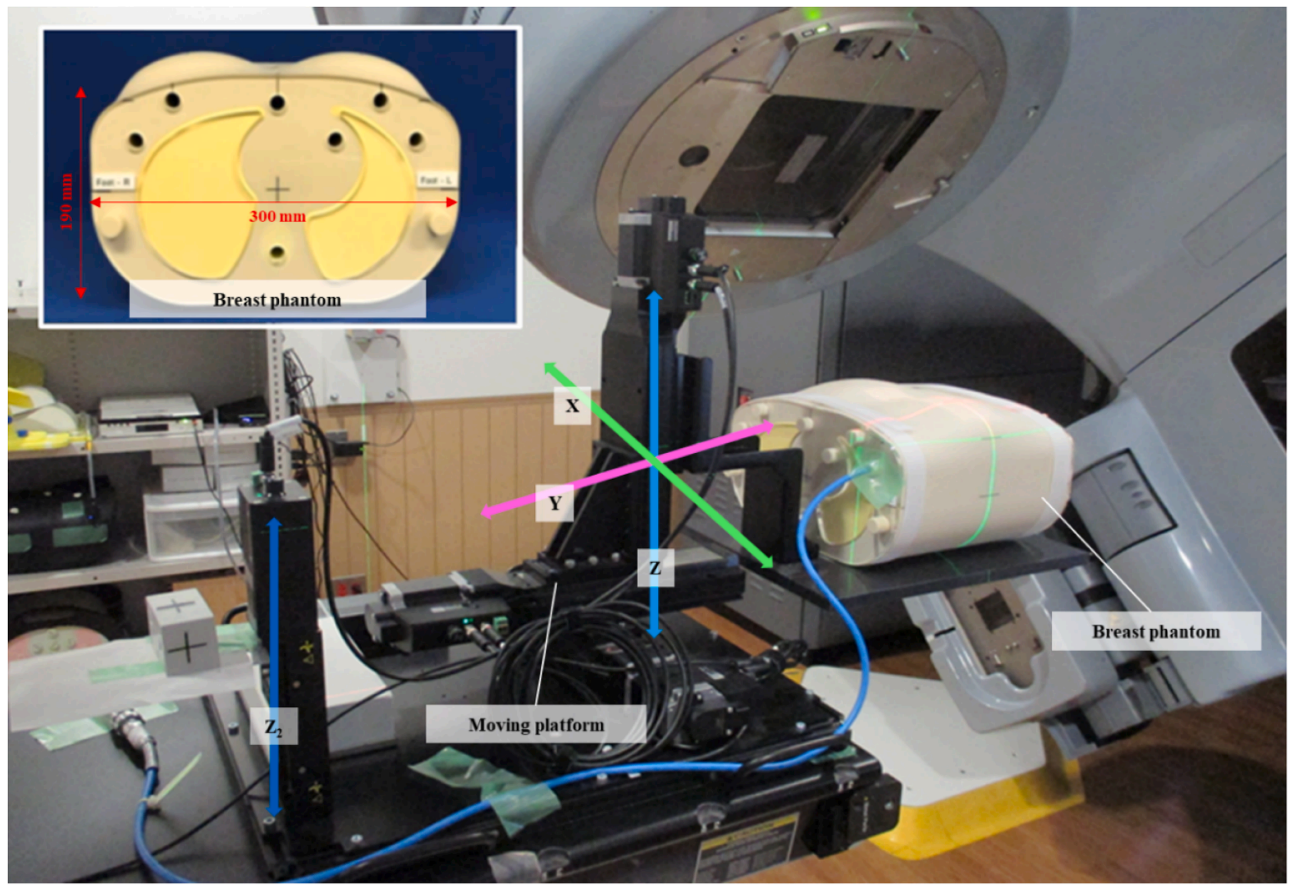

Fig. 1. Measurement setup for the breast phantom and four-axis moving platform.

$2 \% / 2 \mathrm{~mm}$ were also used to assess the gamma passing rates.

\subsection{Statistical analysis}

For the statistical analysis, we employed EZR version 1.37 (Saitama Medical Center, Jichi Medical University, Saitama, Japan), a graphical user interface for R (R Foundation for Statistical Computing, Vienna, Austria) [29]. We conducted a Kruskal-Wallis analysis of variance for the group comparisons as required. In this study, we considered $p<0.05$ statistically significant.

\section{Results}

\subsection{The feasibility of VMAT-APBI for patients with small physiques}

Fig. 2 shows an example of the beam arrangement and dose distributions of the VMAT-APBI plan. Table 3 summarizes the dosimetric parameters of the target and normal tissue, the monitor units, and the beam-on times. Almost all of the dose constraints in DEV_PTV were acceptable, and only one plan was lower in $\mathrm{V}_{27} \mathrm{~Gy}$. The homogeneity index for all plans was within 0.1. The mean \pm standard deviation (SD) of the $\mathrm{V}_{50 \%}$ and $\mathrm{V}_{100 \%}$ values for the uninvolved breast were $20.8 \pm$ $7.3 \%$ and $0.8 \pm 0.4 \%$, respectively.

Fig. 3a shows the mean dose-volume histograms for the 20 plans in all target organs and OARs. The mean or individual dose-volume histograms in the uninvolved breast and heart are shown in Fig. 3b and c; these OARs showed different histograms for each of the patients. The uninvolved breast was displayed separately on the right and left sides because it was difficult to distinguish between the overlapping line plots. In Fig. 3b and c, the patients whose plans were developed by different medical physicists (solid and dot line) are paired with the same color.

\subsubsection{Analysis of the breast respiratory motion}

The mean $( \pm \mathrm{SD})$ respiratory amplitude for the 50 patients in the lateral, longitudinal, and vertical directions was $0.05 \pm 0.06 \mathrm{~cm}, 0.17 \pm$ $0.13 \mathrm{~cm}$, and $0.23 \pm 0.18 \mathrm{~cm}$, respectively, with a respiratory period of $4.5 \pm 1.5 \mathrm{~s}^{1}$. Detailed information is shown in supplementary Table 1 . Fig. 4 shows an example of small, medium, and large amplitude respiratory motion trajectories for the specific patients' breasts. Note that the amplitude was calculated as the vertical distance from peak to trough for each cycle. The respiratory motion in the vertical direction was larger than in other directions, and the patients who had large vertical respiratory motion also tended to have large longitudinal respiratory motion. The percentage of patients with a vertical amplitude $<0.2 \mathrm{~mm}, 0.2-0.3 \mathrm{~mm}, 0.31-0.5 \mathrm{~mm}$, and $>0.5 \mathrm{~mm}$ was $54 \%, 26 \%, 8 \%$, and $12 \%$, respectively (Supplementary Fig. 1).

\subsubsection{Dosimetric verification of VMAT-APBI QA}

Using the gamma analysis criteria of $3 \% / 2 \mathrm{~mm}$, the mean $( \pm \mathrm{SD})$ [range] passing rates was $99.7 \pm 0.4 \%[98.4 \%-100.0 \%]$ for the 20 VMAT-APBI plans measured by the Delta ${ }^{4}$ phantom under static conditions. Moreover, the mean $( \pm \mathrm{SD})$ [range] passing rate was $99.9 \pm 0.1 \%$ [99.5\%-100.0\%] for $3 \% / 3 \mathrm{~mm}$ criteria and $98.9 \pm 0.9 \%$ [96.5\%$100.0 \%$ ] for $2 \% / 2 \mathrm{~mm}$ criteria.

Fig. 5a shows the comparison of the mean relative dose difference between the measured dose and the calculated dose with the ionization chamber at the isocenter. Fig. $5 \mathrm{~b}$ compares the mean relative dose difference between the measured dose under respiratory and static conditions. The 10 VMAT-APBI plans selected for measurement and the three respiratory waveforms are shown in Figs. 2 and 4. All dose differences were within $\pm 3 \%$ regardless of whether they were measured with respiratory motion. As shown in Fig. 5b, there was less of a dose difference with respect to the static condition among measurement conditions in the same patient, but a larger SD was observed for the measurement under large respiratory motion.

The dose distribution measured using radiochromic films were compared to the corresponding calculated dose distributions. Fig. 6 shows boxplots of gamma passing rates due to different measurement conditions, its numerical values were tabulated in Table 4 . The gamma passing rates with $3 \% / 2 \mathrm{~mm}$ criteria for each patient is shown in supplementary Fig. 3. Using the gamma analysis criteria of $3 \% / 2 \mathrm{~mm}$, the mean $( \pm \mathrm{SD})$ gamma passing rates for the static, small, medium, and large amplitude measurements were $95.0 \pm 2.0 \%, 93.3 \pm 3.3 \%, 92.1 \pm$ $6.2 \%$ and $84.8 \pm 7.8 \%$, respectively. The gamma passing rates decreased as the amplitude increased from static to large amplitude, and there was a tendency for a larger SD for the measurements under large respiratory 

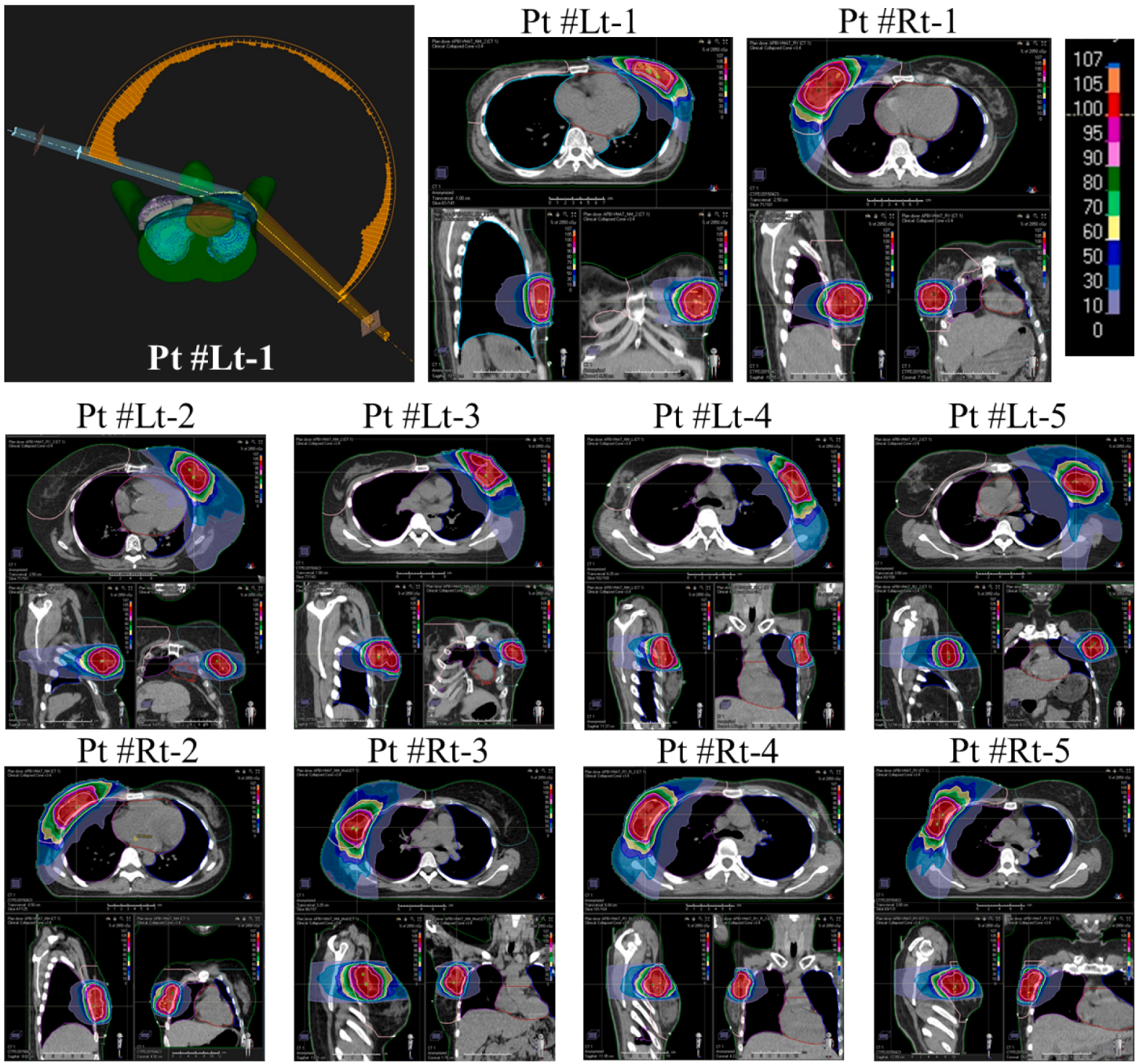

Fig. 2. Example of beam arrangement and dose distributions.

motion. The gamma passing rates for large amplitude measurements were significantly lower than for the other amplitudes $(p<0.001)$, regardless of the acceptance criteria. In certain plans, the results of medium amplitude were better than that of static or small amplitude, but there were no statistically significant values among those of static conditions, small, and medium amplitudes. Fig. 7 shows an example of the calculated and measured dose distribution and a comparison of gamma passing rates with $3 \% / 2 \mathrm{~mm}$ criteria under different respiratory conditions.

\section{Discussion}

In this study, we first evaluated the feasibility of VMAT-APBI for Asian patients with a small physique and small breasts. Based on an analysis of breast respiratory motion in a large population of patients with breast cancer, we performed dosimetric evaluation for VMAT-APBI using a 3D dose verification system, ionization chamber, and radiochromic film.

The mean BMI for the 10 patients who plans were generated by VMAT-APBI was $24.3 \mathrm{~kg} / \mathrm{m}^{2}\left[18.8-35.2 \mathrm{~kg} / \mathrm{m}^{2}\right]$, and most patients were included in the "underweight" or "normal weight" category [30]. When BMI was included as one of the physique indexes, we selected patients with a small physique (compared with European and or North American patients) [31]. However, when we evaluated the dosimetric parameters for the target and OARs, these values were clinically acceptable compared with the constraints set by previous studies [6-8,22,23].

APBI-eligible patients are expected to have excellent long-term cancer-specific survival. Therefore, the risk of toxicity and secondary malignancies needs to be reduced by avoiding the irradiation of healthy organs as much as possible. The dose constraint of the ipsilateral lung was referenced in a previous report that used 3D-comformal radiotherapy (3DCRT) [22], and we were able to significantly reduce the dose using VMAT. Quirk et al. presented more stringent recommendations $\left(\mathrm{V}_{10 \%}<15 \%\right.$ and $\left.\mathrm{V}_{30 \%}<5 \%\right)$ in five-field IMRT [23]. On introduction of VMAT, most of the plans have achieved not only our dose constraints but also Quirk's recommendations. Therefore, dose constraint values of ipsilateral lung should be set more strictly and they could be improved as follows: $\mathrm{V}_{20 \mathrm{~Gy}}<1 \%, \mathrm{~V}_{10 \mathrm{~Gy}}<4 \%$, and $\mathrm{V}_{5 \mathrm{~Gy}}<9 \%$. These recommendations were derived using the dose cutoffs that would be satisfied by $2 \mathrm{SD}$ above the mean of the patients planned.

VMAT plans generally create steep dose gradients and reduce the dose to OARs but increase the low-dose region. A low-dose index such as $\mathrm{V}_{1.5 \mathrm{~Gy}}$ (or $1 \mathrm{~Gy}$ ) is generally adopted for the contralateral lung and heart in APBI and employed to evaluate the out-of-field dose distribution. The 
Table 3

Dosimetric parameters for the 20 plans.

\begin{tabular}{|c|c|c|}
\hline \multirow[t]{2}{*}{ Variables } & \multicolumn{2}{|c|}{ Dosimetric parameters } \\
\hline & Mean \pm SD & Range \\
\hline \multicolumn{3}{|l|}{ DEV_PTV } \\
\hline $\mathrm{V}_{28.5}$ Gy & $95.1 \pm 0.3$ & 94.9-96.1 \\
\hline $\mathrm{V}_{27 \text { Gy }}$ & $99.6 \pm 0.3$ & $98.6-99.9$ \\
\hline $\mathrm{D}_{2 \%}$ & $30.1 \pm 0.1$ & $30.0-30.3$ \\
\hline $\mathrm{D}_{98 \%}$ & $28.1 \pm 0.1$ & $27.9-28.2$ \\
\hline Homogeneity index & $0.068 \pm 0.004$ & $0.059-0.076$ \\
\hline \multicolumn{3}{|l|}{ Uninvolved breast } \\
\hline $\mathrm{V}_{50 \%}$ & $20.8 \pm 7.3$ & $12.4-40.2$ \\
\hline $\mathrm{V}_{100 \%}$ & $0.8 \pm 0.4$ & $0.2-1.9$ \\
\hline \multicolumn{3}{|l|}{ Ipsilateral breast } \\
\hline $\mathrm{V}_{50 \%}$ & $28.7 \pm 8.1$ & $17.6-48.2$ \\
\hline \multicolumn{3}{|l|}{ Contralateral breast } \\
\hline $\mathrm{D}_{2 \mathrm{cc}}$ & $0.9 \pm 0.2$ & $0.4-1.1$ \\
\hline \multicolumn{3}{|l|}{ Ipsilateral lung } \\
\hline $\mathrm{V}_{20 \mathrm{~Gy}}$ & $0.4 \pm 0.3$ & $0.0-1.1$ \\
\hline$V_{10 ~ G y}$ & $1.9 \pm 0.9$ & $0.1-3.5$ \\
\hline $\mathrm{V}_{5 \mathrm{~Gy}}$ & $5.3 \pm 1.8$ & $0.4-8.8$ \\
\hline \multicolumn{3}{|l|}{ Contralateral lung } \\
\hline $\mathrm{V}_{1.5 \mathrm{~Gy}}$ & $0.1 \pm 0.4$ & $0.0-1.5$ \\
\hline \multicolumn{3}{|l|}{ Lungs } \\
\hline $\mathrm{V}_{20 \mathrm{~Gy}}$ & $0.2 \pm 0.2$ & $0.0-0.6$ \\
\hline$V_{10 ~ G y}$ & $1.0 \pm 0.5$ & $0.0-2.0$ \\
\hline$V_{5}$ Gy & $2.7 \pm 1.0$ & $0.2-4.9$ \\
\hline \multicolumn{3}{|l|}{ Heart } \\
\hline $\mathrm{V}_{1.5 \text { Gy }}$ (right) & $2.5 \pm 4.0$ & $0.0-12.1$ \\
\hline $\mathrm{V}_{20 \text { Gy }}$ (left) & $0.0 \pm 0.0$ & $0.0-0.0$ \\
\hline $\mathrm{V}_{5 \text { Gy }}$ (left) & $0.2 \pm 0.4$ & $0.0-1.4$ \\
\hline $\mathrm{V}_{3 \text { Gy }}$ (left) & $1.4 \pm 2.5$ & $0.0-8.1$ \\
\hline $\mathrm{V}_{1.5 \text { Gy }}$ (left) & $8.6 \pm 10.3$ & $0.0-32.1$ \\
\hline Conformity index & $1.02 \pm 0.03$ & $0.98-1.11$ \\
\hline Paddick conformity index & $0.89 \pm 0.02$ & $0.81-0.92$ \\
\hline Gradient index & $3.29 \pm 0.47$ & $2.47-4.02$ \\
\hline Monitor units, MUs & $1598.5 \pm 137.0$ & $1267.0-1797.9$ \\
\hline Beam-on times, s & $173.5 \pm 11.2$ & $150.0-191.0$ \\
\hline
\end{tabular}

value of $\mathrm{D}_{2 \mathrm{cc}}$ (or $2 \%$, max) to contralateral breast was also used to evaluate low-dose area spread. Although these approximate values were within each constraint in this population, there was deviation due to the target position and patient physique. When treating inner area targets with VMAT, it can be difficult to achieve the dose constraint because of low-dose spread. The previous reports with 3DCRT or static-IMRT used $1 \mathrm{~Gy}$ for the contralateral breast [6-8]; however, for the above reason, our institution loosely set the constraint in advance $\left(\mathrm{D}_{2 \mathrm{cc}} \leqq 1.5 \mathrm{~Gy}\right)$. In the case of this study, 3 plans of 2 patients (Lt- 1 and Lt-2) were $\mathrm{D}_{2 \mathrm{cc}}=$ $1.1 \mathrm{~Gy}$ and could not achieved acceptable level of $1 \mathrm{~Gy}$, but our constraint value of additional $0.5 \mathrm{~Gy}$ was considered to have little clinical impact.

Paddick CI for DEV_PTV was calculated and mean $( \pm \mathrm{SD})$ Paddick CI was $0.89 \pm 0.02$ (Table 3). For the 20 plans in this study, the mean $( \pm$ SD) RTOG CI was $1.02 \pm 0.03$, and the volume of the uninvolved breast irradiated by the prescribed dose was $0.8 \pm 0.4 \%[0.2 \%-1.9 \%]$. Therefore, more conformal plans that fit the target were generated. Other studies reported a CI planned by IMRT or VMAT of 1.1-1.2 $[11,23]$, which is comparable to our results. The cause for the lower conformity of the dose distribution in previous reports is 3DCRT planning [11-13], which can be solved by introducing VMAT even for patient with small breasts. Grendarova et al. reported that cosmesis outcomes have not been completely linked to dose conformity, but improving conformity should be a priority [9].

An interesting finding was that the mean breast volume in our cohort was $945.4 \pm 439.1 \mathrm{~cm}^{3}$, much smaller than the $1480 \mathrm{~cm}^{3}$ measured in the previous study [32], and there was large deviation in the $\mathrm{V}_{50 \%}$ value

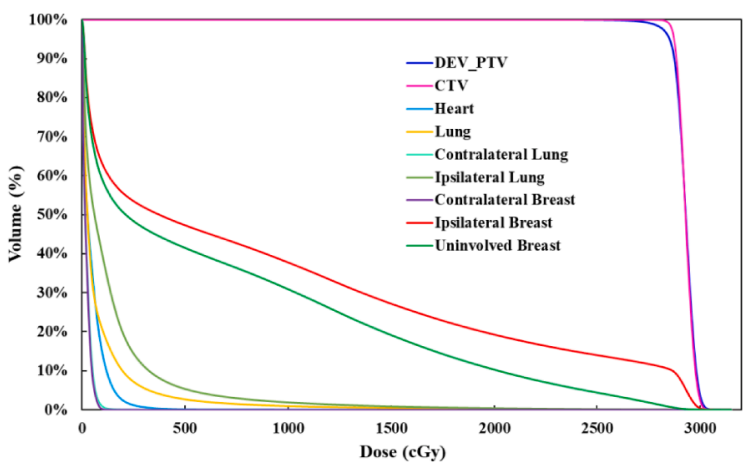

(a) All target and OARs (average for 20 plans)
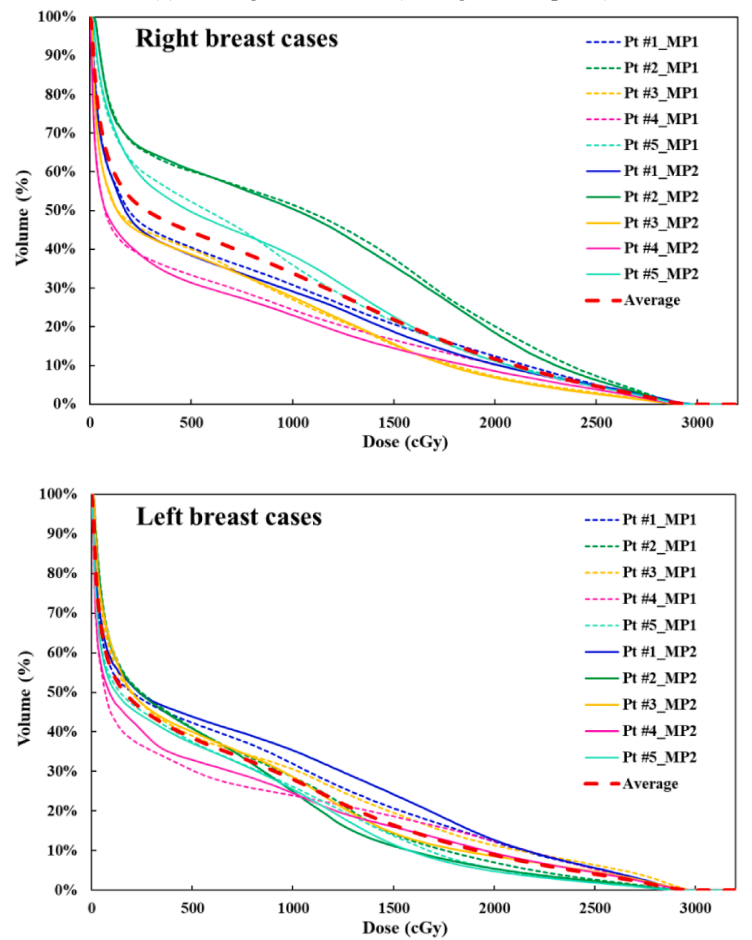

(b) Uninvolved breast (average shows mean of 10 plans for patients with each right and left breast cancer)

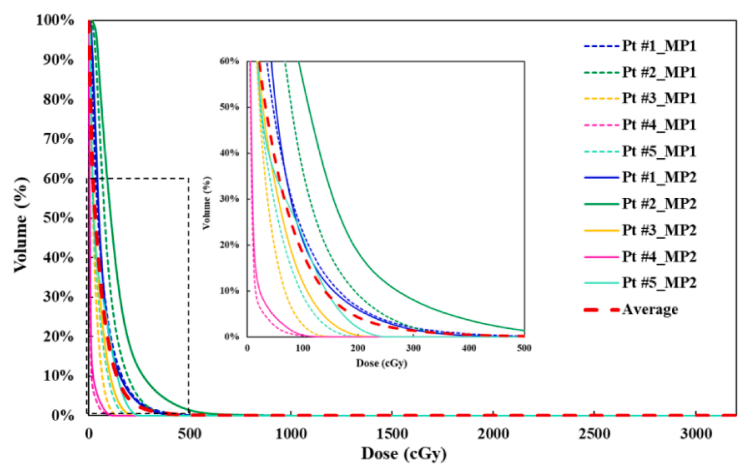

(c) Heart (average shows mean of 10 plans for patients with left-sided breast cancer)

Fig. 3. Average or individual dose-volume histograms in target and OARs. (a) All target and OARs for all patients, (b) Uninvolved breast for patients with right- and left-sided breast cancer, (c) Heart for patients with left-sided breast cancer, the dose-volume histograms between 0 and 500 cGy are superimposed for comparison. Patients whose plans were created by different medical physicists [dot and solid line (MP1 or MP2)] are paired with the same color. Abbreviations: Pt, patient; MP, medical physicist. 


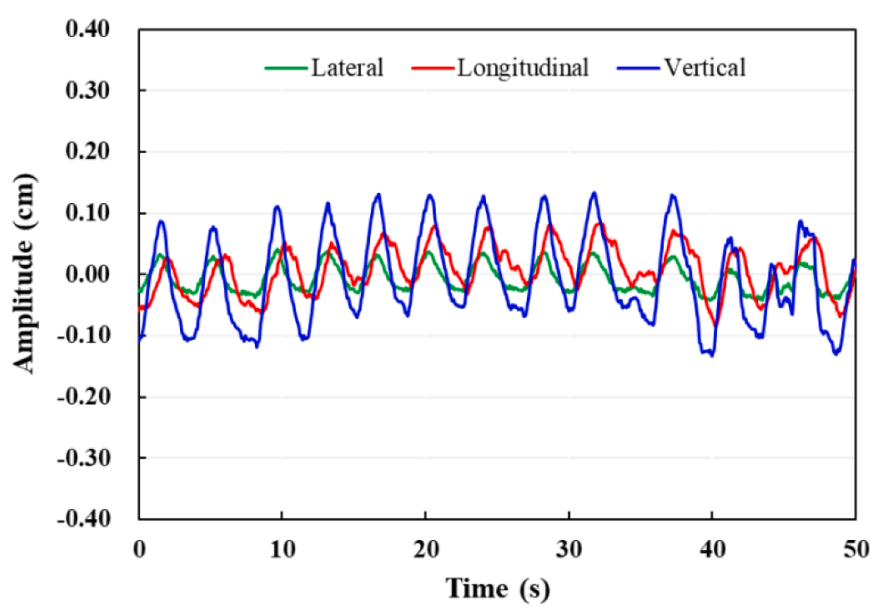

(a) Small respiratory amplitude [Amplitude: lateral $=0.07 \mathrm{~cm}$, longitudinal $=0.11 \mathrm{~cm}$, vertical $=0.20 \mathrm{~cm}$ ]

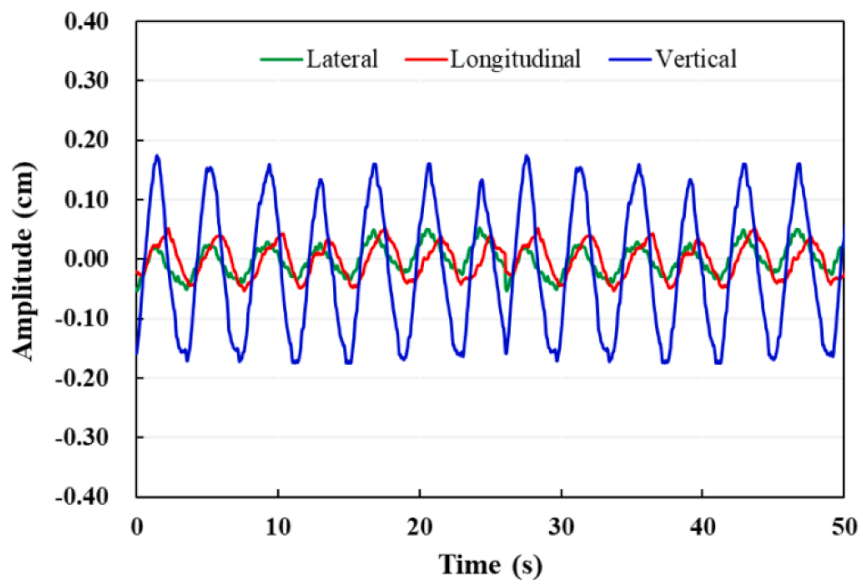

(b) Medium respiratory amplitude [Amplitude: lateral $=0.08 \mathrm{~cm}$, longitudinal $=0.09 \mathrm{~cm}$, vertical $=0.35$

$\mathrm{cm}]$

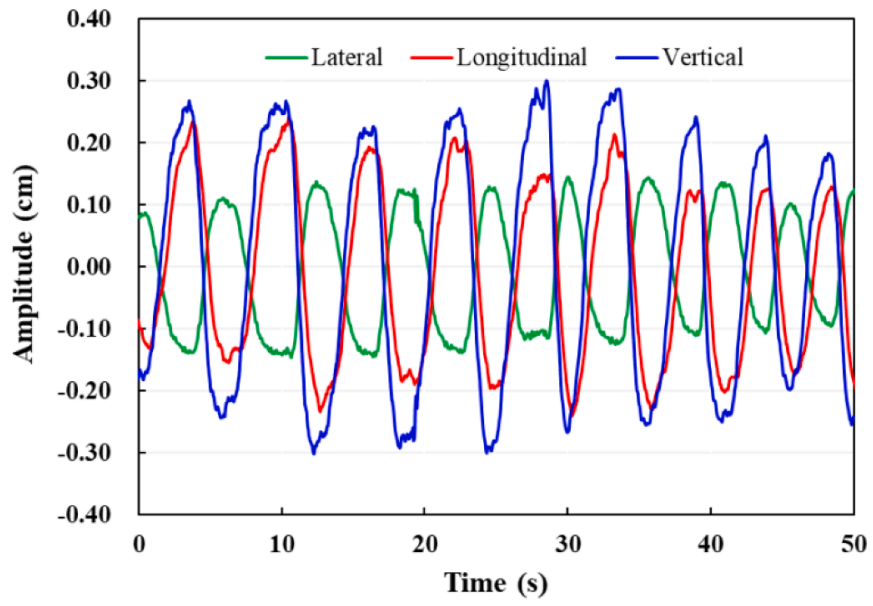

(c) Large respiratory amplitude [Amplitude: lateral $=0.26 \mathrm{~cm}$, longitudinal $=0.38 \mathrm{~cm}$, vertical $=0.53 \mathrm{~cm}$ ]

Fig. 4. Small, medium and large amplitude trajectories of the respiratory motion of the patients' breasts.

among patients for the uninvolved or ipsilateral breast. Previous studies have reported the relationship between dosimetric parameters (the maximum point dose, the irradiated breast volume to normal breast volume) and cosmetic outcomes [32,33]. Livi et al. reported that breast cosmesis was related to the volume of breast that received $50 \%$ of the prescribed dose and that better dose homogeneity and sparing of the uninvolved breast can be achieved by introducing IMRT, which might have a favorable effect in reducing the occurrence of adverse events [8]. 


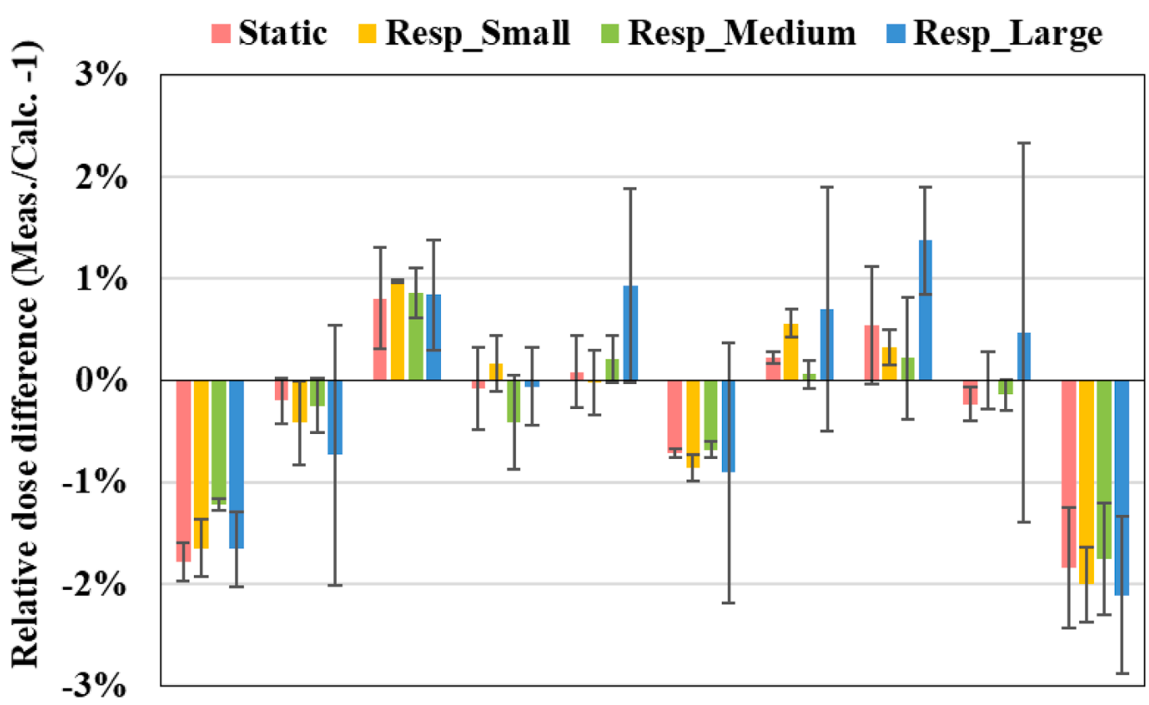

Lt-1 Lt-2 Lt-3 Lt-4 $\begin{aligned} & \text { Lt-5 Rt-1 Rt-2 Rt-3 Rt-4 Rt-5 } \\ & \text { Patient No. }\end{aligned}$
Fig. 5. (a) Comparison of the mean relative dose difference between the measured dose and the calculated dose with the ionization chamber at the isocenter. (b) Comparison of the mean relative dose difference between the measured dose under respiratory conditions and the static condition. The legend of "Static", "Resp_small", "Resp_Medium", and "Resp_Large" indicates that measured under the static condition and three respiratory motion conditions. Error bar shows 1 standard deviation.

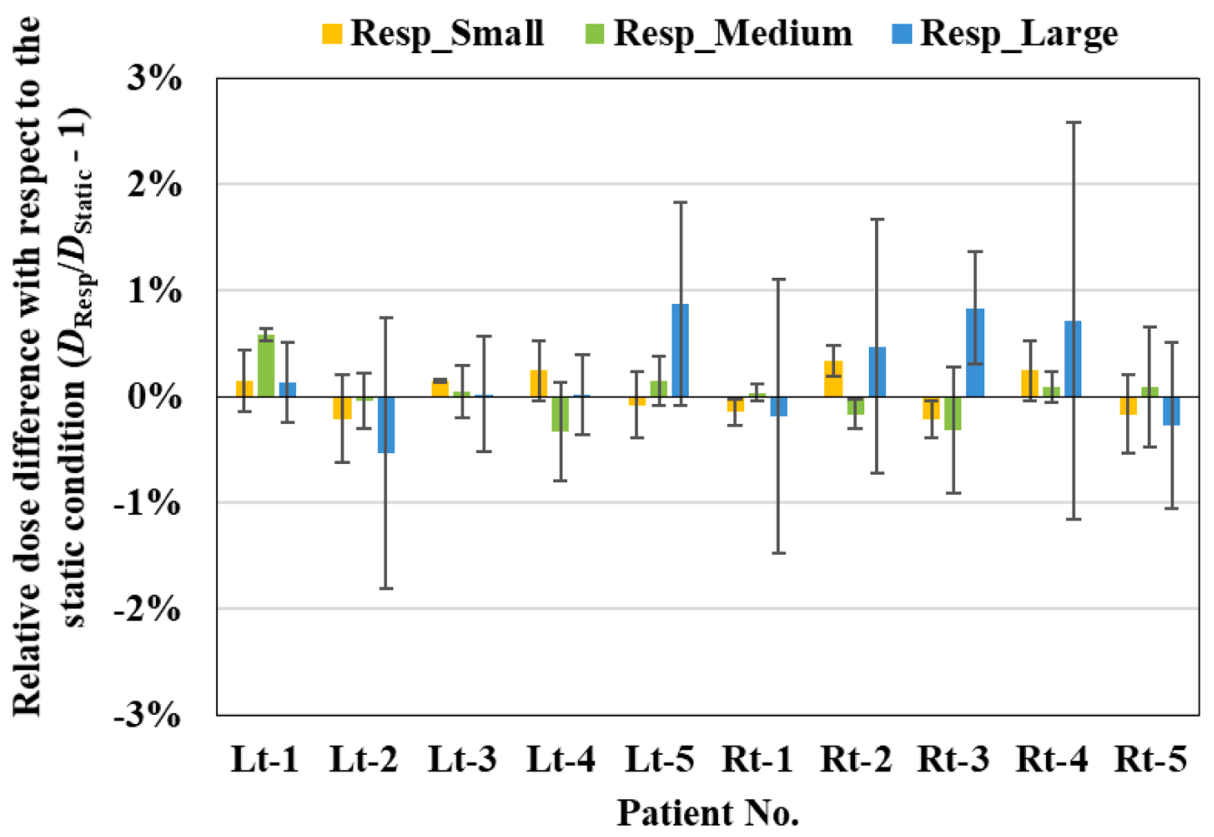

(b)

Minimizing the irradiation of healthy breast tissue is therefore essential. For patients with small breasts, the volume ratio of DEV_PTV to ipsilateral breast tends to be higher and might be disadvantageous compared with patients with large breasts. For example, one of the study patients with right-sided breast cancer (Rt-2) had a relatively higher volume ratio than the other patients. Consequently, the V50\% value resulted in a different histogram (Fig. 3b). An additional analysis was performed to clarified relationship between ipsilateral breast $\mathrm{V}_{50 \%}$ and the ratio of DEV_PTV to ipsilateral breast. (Supplementary Fig. 2) The Pearson correlation coefficient was used for correlational analyses and we found a strong correlation $(r=0.8397)$. The uniform constraint regardless of breast size might be too strict for patients with an unfavorable physique, but this concern requires further investigation and verification in a large patient cohort.

Accurate determination, management and control of the magnitude of respiratory motion in radiation therapy is an important aspect of modern treatment planning and delivery, as described in AAPM Task Group 76 and C. Cavedon $[17,34]$. This study analyzed the respiratory motion of the breasts using tracking data obtained by an external infrared marker, which is an appropriate marker for approximating breast motion. Quirk et al. observed a respiratory amplitude of $0.2 \pm 0.2$ $\mathrm{cm}$ in the vertical direction [18], a finding consistent with our results of 


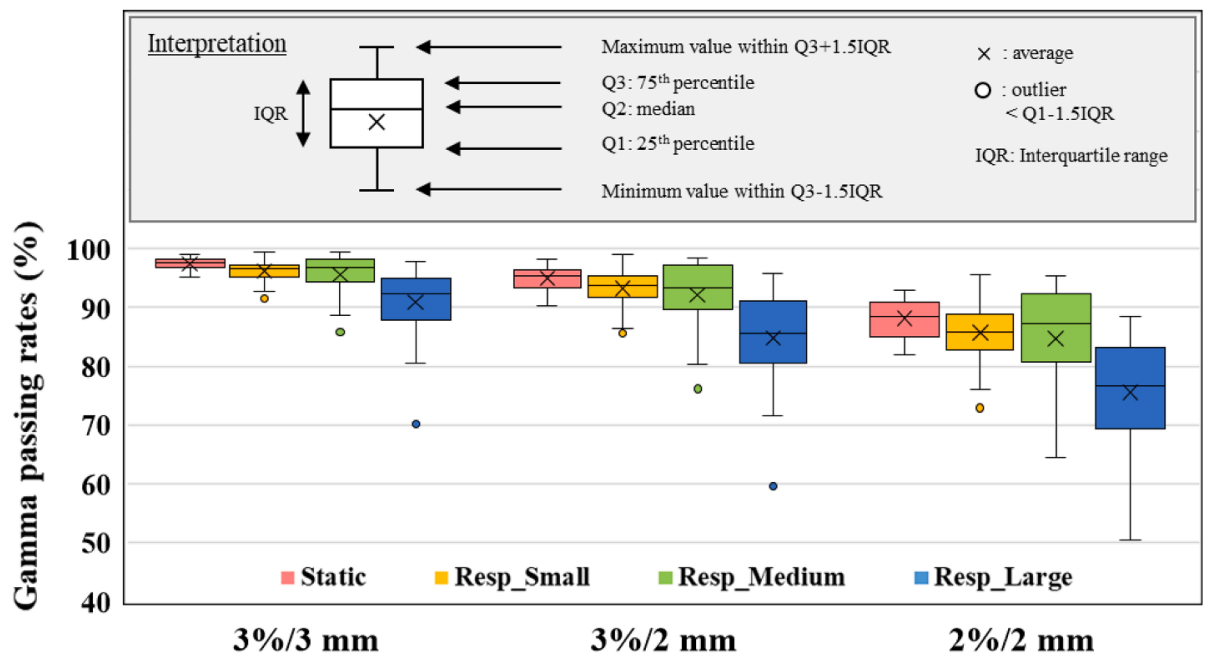

Fig. 6. Boxplots of gamma passing rates due to difference of measurement conditions that measured by radiochromic films. Acceptance criteria of $3 \% / 3 \mathrm{~mm}, 3 \% / 2$ $\mathrm{mm}$, and $2 \% / 2 \mathrm{~mm}$ were used.

Table 4

Average gamma passing rate with standard deviation in global gamma analysis of radiochromic film.

\begin{tabular}{|c|c|c|c|c|}
\hline \multirow{2}{*}{$\begin{array}{l}\text { Acceptance } \\
\text { criteria }\end{array}$} & \multicolumn{4}{|c|}{ Measurement condition with static or respiratory movement } \\
\hline & Static & $\begin{array}{l}\text { Small } \\
\text { amplitude }\end{array}$ & $\begin{array}{l}\text { Medium } \\
\text { amplitude }\end{array}$ & $\begin{array}{l}\text { Large } \\
\text { amplitude }\end{array}$ \\
\hline $3 \% / 3 \mathrm{~mm}$ & $\begin{array}{l}97.4 \pm \\
1.0 \%\end{array}$ & $96.2 \pm 1.8 \%$ & $95.5 \pm 3.7 \%$ & $90.8 \pm 5.7 \%$ \\
\hline $3 \% / 2 \mathrm{~mm}$ & $\begin{array}{l}95.0 \pm \\
2.0 \%\end{array}$ & $93.3 \pm 3.3 \%$ & $92.1 \pm 6.2 \%$ & $84.8 \pm 7.8 \%$ \\
\hline $2 \% / 2 \mathrm{~mm}$ & $\begin{array}{l}88.2 \pm \\
3.1 \%\end{array}$ & $85.7 \pm 5.2 \%$ & $84.7 \pm 8.8 \%$ & $75.6 \pm 7.8 \%$ \\
\hline
\end{tabular}

$0.23 \pm 0.18 \mathrm{~cm}$; however, the authors' results showed only one direction and targeted healthy volunteers who were under less stress than those undergoing radiotherapy. Kinoshita et al. investigated respiratory amplitude by monitoring the location of the gold marker that was placed on side of the breast with a fluoroscopic tracking system, the mean $( \pm \mathrm{SD})$ range of motion was $1.0 \pm 0.6 \mathrm{~mm}, 1.3 \pm 0.5 \mathrm{~mm}$, and $2.6 \pm 1.4$ $\mathrm{mm}$ for the lateral, longitudinal, and vertical directions, respectively
[35]. The respiratory motion for women with breast cancer is mainly vertical and longitudinal, and the amplitude is smaller than that of patients with lung and abdominal cancer [17]. We therefore focused on the dosimetric evaluation due to the relatively smaller amplitude (<approximately $5 \mathrm{~mm}$ ); however, the evaluation would apply to most patients. Investigating the breast respiratory motion in this large number of patients is applicable not only to APBI but also to whole breast irradiation and chest wall irradiation using IMRT.

Radiotherapy with respiratory motion management requires an adequate moving phantom for quality assurance [17]. Certain commercial systems provide solutions to reproduce tumor motion; however, in some cases, tumor and chest motion are only partially implemented. For this reason, we employed 4-axis moving platform (4D-KAMUI) to reproduce breast motion that move primarily in superior-inferior and anterior-posterior directions. Furthermore, several phantoms have been designed that are more similar the human systems, such as the IMRT torso (Computerized Imaging Reference System Inc., Norfolk, VA, USA), Alderson (Radiological Support Devices Inc., Long Beach, CA, US), and Rault's breast phantoms [36]. However, the shape of certain phantoms is simplified and does not closely resemble the size or shape of Asian women's breasts. Pallotta et al. mentioned the need to implement a
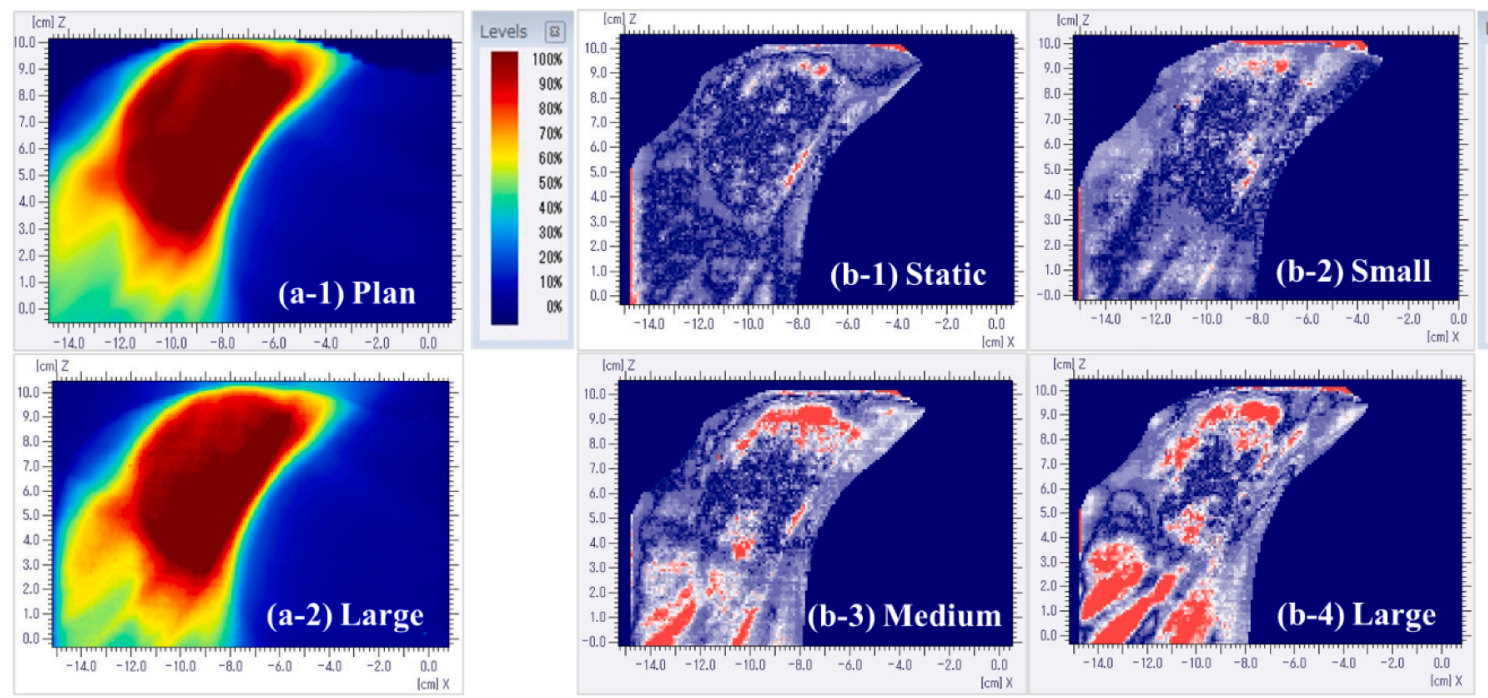

Fig. 7. Example of calculated and measured dose distribution (left) and comparison of gamma passing rates by different respiratory conditions (right) in patient of Rt-5. Gamma pass analysis with $3 \% / 2 \mathrm{~mm}$ criteria. 
realistic human phantom including internal anatomical details and platforms that reproduce target movement [37]. Therefore, we measured using an original breast phantom modeling the average Asian woman's body shape.

The gamma analysis is widely used to quantitatively evaluate the delivered dose distribution. The dose comparisons were performed using the following three criteria: $3 \% / 3 \mathrm{~mm}, 3 \% / 2 \mathrm{~mm}$, and $2 \% / 2 \mathrm{~mm}$. The influence of respiratory motion is clear when looking at the resulting gamma passing rates, and there was a significant difference in the dosimetric analysis between larger and other smaller respiration amplitudes regardless of acceptance criteria. As shown in Fig. 7 of the film measurement results, we observed numerous unintended dose distributions outside the target as the amplitude increased. Streak dose errors outside the target also became more noticeable as the amplitude increased. For partial arc VMAT-APBI, the majority of the dose was delivered by tangential beams, and the rotational speed at that time was extremely slow (approximately $1 \mathrm{deg} / \mathrm{s}$ ). The dose errors were therefore greater as the amplitude increased because the high doses were delivered when the respiratory motion direction (vertical) matched the gantry rotation direction. The main cause of these dose errors could be the interplay effect and blurring effect of dose distribution [14,38]. On the other hand, the dose difference measured by ionization chamber was small among the respiratory conditions. The point dose measurement was performed at the center of the PTV, and these areas had no significant differences between the measurement conditions when looking at the gamma passing rate results (Fig. 7). However, the dose deviation was larger as the amplitude increased, which might be caused by the interplay effect between the multileaf collimator motion and the intrafractional target motion. Bortfeld et al. and George et al. reported that the interplay effect is minimal compared with the blurring effect of the dose distribution resulting from respiratory motion $[14,38]$.

To our knowledge, this is first study to evaluate the influence of respiratory motion on dose distribution for VMAT-APBI. The introduction of VMAT to APBI had not been reported much, and the dose coverage of PTV is ensured by setting a sufficient internal margin in case the APBI uses 3DCRT. The influence of interplay effect due to respiratory motion is generally reduced for multiple fractions. [14,15,39] Respiratory motion might have a negligible effect on treatment with 10 fractions due to the average effect, even if it is IMRT-APBI. This average effect only applies if the initial breathing phase is evenly distributed. Therefore, it might not be possible to expect the average effect with low number of fractions because the probability of starting the treatment in the same phase for each fraction is larger. [40] In VMAT-APBI with five fractions, we recommend that considering respiratory motion management techniques (such as breath holding and shallow breathing) if a $>5$ $\mathrm{mm}$ range of motion is observed.

\section{Conclusions}

We evaluated the feasibility of using VMAT-APBI for Asian patients who have a small physique and small breasts and found comparable dose distributions to those of previous reports. We investigated the amplitude of the respiratory motion during VMAT-APBI delivery that significantly affects dose distribution. In our results, we observed numerous unintended dose distributions around the target as the amplitude increased. Therefore, it is recommended to consider respiratory motion management techniques, such as breath-hold and shallow breathing, if the amplitude is $>5 \mathrm{~mm}$.

\section{Acknowledgement}

This study was supported in part by St. Luke's Breast Charity Fund.

\section{Declaration of Competing Interest}

The authors declare that they have no known competing financial interests or personal relationships that could have appeared to influence the work reported in this paper.

\section{Appendix A. Supplementary data}

Supplementary data to this article can be found online at https://doi. org/10.1016/j.ejmp.2020.09.024.

\section{References}

[1] Bray F, Ferlay J, Soerjomataram I, Siegel RL, Torre LA, Jemal A. Global cancer statistics 2018: GLOBOCAN estimates of incidence and mortality worldwide for 36 cancers in 185 countries. CA Cancer J Clin 2018;68(6):394-424. https://doi.org/ $10.3322 /$ caac. 21492.

[2] Early Breast Cancer Trialists' Collaborative Group (EBCTCG). Effect of radiotherapy after breast-conserving surgery on 10-year recurrence and 15-year breast cancer death: meta-analysis of individual patient data for 10801 women in 17 randomised trials. The Lancet 2011;378:1707-16. https://doi. org/10.1016/S0140-6736(11)61629-2.

[3] Early Breast Cancer Trialists' Collaborative Group (EBCTCG). Effects of radiotherapy and of differences in the extent of surgery for early breast cancer on local recurrence and 15-year survival: an overview of the randomised trials. The Lancet 2005;366:2087-106. https://doi.org/10.1016/S0140-6736(05)67887-7.

[4] Olivotto IA, Whelan TJ, Parpia S, Kim D-H, Berrang T, Truong PT, et al. Interim cosmetic and toxicity results from RAPID: a randomized trial of accelerated partial breast irradiation using three-dimensional conformal external beam radiation therapy. J Clin Oncol 2013;31(32):4038-45. https://doi.org/10.1200/ JCO 2013.50.5511.

[5] Whelan TJ, Julian JA, Berrang TS, Kim D-H, Germain I, Nichol AM, et al. External beam accelerated partial breast irradiation versus whole breast irradiation after breast conserving surgery in women with ductal carcinoma in situ and nodenegative breast cancer (RAPID): a randomised controlled trial. The Lancet 2019; 394(10215):2165-72. https://doi.org/10.1016/S0140-6736(19)32515-2.

[6] National Surgical Adjuvant Breast Bowel Project (NSABP)/Radiation Therapy Oncology Group (RTOG) NSABP protocol B-39/RTOG protocol 0413. A Randomized Phase III Study of Conventional Whole Breast Irradiation (WBI) Versus Partial Breast Irradiation (PBI) for Women with Stage 0, I, or II Breast Cancer.

[7] Vicini FA, Cecchini RS, White JR, Julian TB, Arthur DW, Rabinovitch RA, et al., Abstract GS4-04: Primary results of NSABP B-39/RTOG 0413 (NRG Oncology): A randomized phase III study of conventional whole breast irradiation (WBI) versus partial breast irradiation (PBI) for women with stage 0 , I, or II breast cancer. The 2018 San Antonio Breast Cancer Symposium. San Antonio, TX: AACR; Cancer Res; 2018.

[8] Livi L, Meattini I, Marrazzo L, Simontacchi G, Pallotta S, Saieva C, et al. Accelerated partial breast irradiation using intensity-modulated radiotherapy versus whole breast irradiation: 5-year survival analysis of a phase 3 randomised controlled trial. Eur J Cancer 2015;51(4):451-63. https://doi.org/10.1016/j. ejca. 2014.12.013.

[9] Grendarova P, Roumeliotis M, Quirk S, Lesiuk M, Craighead P, Liu HW, et al. Oneyear cosmesis and fibrosis from ACCEL: Accelerated partial breast irradiation (APBI) using 27 gy in 5 daily fractions. Pract Radiat Oncol 2019;9:e457-64. https://doi.org/10.1016/j.prro.2019.04.002.

[10] Ono Y, Yoshimura M, Hirata K, Ono T, Hirashima H, Mukumoto N, et al. Dosimetric advantages afforded by a new irradiation technique, Dynamic WaveArc, used for accelerated partial breast irradiation. Phys Med 2018;48: 103-10. https://doi.org/10.1016/j.ejmp.2018.03.015.

[11] Qiu J-J, Chang Z, Horton JK, Wu Q-R, Yoo S, Yin F-F. Dosimetric comparison of 3D conformal, IMRT, and V-MAT techniques for accelerated partial-breast irradiation (APBI). Med Dosim 2014;39(2):152-8. https://doi.org/10.1016/j. meddos.2013.12.001.

[12] Kosaka Y, Mitsumori M, Yamauchi C, Narita Y, Hiraoka M. Feasibility of accelerated partial breast irradiation using three-dimensional conformal radiation therapy for Japanese women: a theoretical plan using six patients' CT data. Breast Cancer 2008;15(1):108-14. https://doi.org/10.1007/s12282-007-0013-0.

[13] Mitsumori M, Hiraoka M. Current status of accelerated partial breast irradiation. Breast Cancer 2008;15(1):101-7. https://doi.org/10.1007/s12282-007-0012-1.

[14] Bortfeld T, Jiang S, Rietzel E. Effects of motion on the total dose distribution. Semin Radiat Oncol 2004;14(1):41-51. https://doi.org/10.1053/j. semradonc. 2003.10.011.

[15] Bortfeld T, Jokivarsi K, Goitein M, Kung J, Jiang SB. Effects of intra-fraction motion on IMRT dose delivery: statistical analysis and simulation. Phys Med Biol 2002;47: 2203-20. https://doi.org/10.1088/0031-9155/47/13/302.

[16] Netherton T, Li Y, Nitsch P, Shaitelman S, Balter P, Gao S, et al. Interplay effect on a 6-MV flattening-filter-free linear accelerator with high dose rate and fast multileaf collimator motion treating breast and lung phantoms. Med Phys 2018;45(6): 2369-76. https://doi.org/10.1002/mp.12899.

[17] Keall PJ, Mageras GS, Balter JM, Emery RS, Forster KM, Jiang SB, et al. The management of respiratory motion in radiation oncology report of AAPM Task Group 76. Med Phys 2006;33:3874-900. https://doi.org/10.1118/1.2349696.

[18] Quirk S, Becker N, Smith WL. External respiratory motion analysis and statistics for patients and volunteers. J Appl Clin Med Phys 2013;14(2):90-101. https://doi. org/10.1120/jacmp.v14i2.4051. 
[19] Zhao H, Williams N, Poppe M, Sarkar V, Wang B, Rassiah-Szegedi P, et al. Comparison of surface guidance and target matching for image-guided accelerated partial breast irradiation (APBI). Med Phys 2019;46(11):4717-24. https://doi.org/ 10.1002/mp.13816.

[20] Wang CS, Josenhans WT. Contribution of diaphragmatic-abdominal displacement to ventilation in supine man. J Appl Physiol 1971;31(4):576-80. https://doi.org/ 10.1152/jappl.1971.31.4.576.

[21] White J, Tai A, Arthur D, Buchholz T, MacDonald S, Marks L, et al. Breast cancer atlas for radiation therapy planning: consensus definitions. Philadelphia, PA: Radiation Therapy Oncology Group; 2009.

[22] Recht A, Ancukiewicz M, Alm El-Din MA, Lu X-Q, Martin C, Berman SM, et al. Lung dose-volume parameters and the risk of pneumonitis for patients treated with accelerated partial-breast irradiation using three-dimensional conformal radiotherapy. J Clin Oncol 2009;27(24):3887-93. https://doi.org/10.1200/ JCO.2008.20.0121.

[23] Quirk S, Grendarova P, Roumeliotis M. Five-field IMRT class solutions and dosimetric planning guidelines for implementing accelerated partial breast irradiation. Pract Radiat Oncol 2018;8(3):e99-107. https://doi.org/10.1016/j. prro.2017.09.009.

[24] Hodapp N. The ICRU Report 83: prescribing, recording and reporting photon-beam intensity-modulated radiation therapy (IMRT). Strahlenther Onkol 2012;188:97-9. https://doi.org/10.1007/s00066-011-0015-x.

[25] Shaw E, Kline R, Gillin M, Souhami L, Hirschfeld A, Dinapoli R, et al. Radiation therapy oncology group: radiosurgery quality assurance guidelines. Int $\mathrm{J}$ Radiat Oncol Biol Phys 1993;27(5):1231-9. https://doi.org/10.1016/0360-3016(93) 90548-a.

[26] Cao T, Dai Z, Ding Z, Li W, Quan H. Analysis of different evaluation indexes for prostate stereotactic body radiation therapy plans: conformity index, homogeneity index and gradient index. Prec Radiat Oncol 2019;3(3):72-9. https://doi.org/ 10.1002/pro6.1072.

[27] Paddick I, Lippitz B. A simple dose gradient measurement tool to complement the conformity index. J Neurosurg 2006;105(suppl.):194-201. https://doi.org/ 10.3171/sup.2006.105.7.194.

[28] Kito S, Karasawa K, Nihei K, Motoyoshi K, Hashimoto T, Furuya T, et al. The development of new dynamic moving phantom with four axis drives. Int $\mathrm{J}$ Radiat Oncol Biol Phys 2015;93(3):E596. https://doi.org/10.1016/j.ijrobp.2015.07.2069.

[29] Kanda Y. Investigation of the freely available easy-to-use software 'EZR' for medical statistics. Bone Marrow Transplant 2013;48(3):452-8. https://doi.org/ 10.1038/bmt.2012.244.
[30] WHO/Europe Nutrition body mass index BMI. <http://www.euro.who.int/en/hea lth-topics/disease-prevention/nutrition/a-healthy-lifestyle/body-mass-index-bmi> (accessed 26 April 2020).

[31] NCD Risk Factor Collaboration (NCD-RisC). Trends in adult body-mass index in 200 countries from 1975 to 2014: a pooled analysis of 1698 population-based measurement studies with 19.2 million participants. Lancet 2016;387:1377-96. https://doi.org/10.1016/S0140-6736(16)30054-X.

[32] Quirk S, Grendarova P, Craighead P, Phan T, Lesiuk M, Pinilla J, et al. Results of the ACCEL trial: dosimetry in accelerated partial breast irradiation. Radiother Oncol 2020;147:50-5. https://doi.org/10.1016/j.radonc.2020.03.004.

[33] Leonard KL, Hepel JT, Hiatt JR, Dipetrillo TA, Price LL, Wazer DE. The effect of dose-volume parameters and interfraction interval on cosmetic outcome and toxicity after 3-dimensional conformal accelerated partial breast irradiation. Int $\mathrm{J}$ Radiat Oncol Biol Phys 2013;85(3):623-9. https://doi.org/10.1016/j. ijrobp.2012.06.052

[34] Cavedon C. Real-time control of respiratory motion: beyond radiation therapy. Phys Med 2019;66:104-12. https://doi.org/10.1016/j.ejmp.2019.09.241.

[35] Kinoshita R, Shimizu S, Taguchi H, Katoh N, Fujino M, Onimaru R, et al. Threedimensional intrafractional motion of breast during tangential breast irradiation monitored with high-sampling frequency using a real-time tumor-tracking radiotherapy system. Int J Radiat Oncol Biol Phys 2008;70(3):931-4. https://doi. org/10.1016/j.ijrobp.2007.10.003.

[36] George R, Keall PJ, Kini VR, Vedam SS, Siebers JV, Wu Q, et al. Quantifying the effect of intrafraction motion during breast IMRT planning and dose delivery. Med Phys 2003;30(4):552-62. https://doi.org/10.1118/1.1543151.

[37] Rault E, Lacornerie T, Dang H-P, Crop F, Lartigau E, Reynaert N, et al. Accelerated partial breast irradiation using robotic radiotherapy: a dosimetric comparison with tomotherapy and three-dimensional conformal radiotherapy. Radiat Oncol 2016; 11(1). https://doi.org/10.1186/s13014-016-0607-9.

[38] Pallotta S, Calusi S, Foggi L, Lisci R, Masi L, Marrazzo L, et al. ADAM: a breathing phantom for lung SBRT quality assurance. Phys Med 2018;49:147-55. https://doi. org/10.1016/j.ejmp.2017.07.004.

[39] Duan J, Shen S, Fiveash JB, Popple RA, Brezovich IA. Dosimetric and radiobiological impact of dose fractionation on respiratory motion induced IMRT delivery errors: a volumetric dose measurement study: dose fractionation impact on motion induced IMRT errors. Med Phys 2006;33(5):1380-7. https://doi.org/ 10.1118/1.2192908.

[40] Edvardsson A, Nordstrom F, Ceberg C, Ceberg S. Motion induced interplay effects for VMAT radiotherap. Phys Med Biol. 2018;63. https://doi.org/10.1088/13616560/aab957. 